\title{
A Researchon the Performance of the Diploma Students in theLateral Entry Scheme of Engineering Degree Program
}

\author{
G. S. Nandakumar, L. M. Palanivelu
}

\begin{abstract}
The diploma holders' performance in engineering education through the lateral entry scheme has been found far below the standard of the regular students who join after higher secondary education. Ideally both should fare equally at graduation. There are lacunae in the process of Lateral EntryLE) admission and few other parameters that pull down the efficiency of the LE standards. We have taken up this study to identify the parameters that cause the $L E$ to cave in.
\end{abstract}

Index Terms - Engineering education, Lateral entry scheme.

\section{INTRODUCTION}

It is now a well recognized fact that there is an overproduction of engineering graduates with less than 25percent of them absorbed in meaningful engineering jobs. Most of these graduates lack in skills required by the recruiting companies. These companies have to bridge the skill gap by scheduling appropriate training program to make them productive. This indirectly points to the lack of expected quality in our engineering education. To enhance the volume of engineering graduates produced and to provide a fair chance for the bright diploma holders to obtain a degree, there is a provision to admit these diploma holders to enter the four year engineering degree programs at the beginning of second year. In this paper we have analyzed the impact of this lateral entry (LE) scheme on the quality of engineering education and the career prospects for these students.

Normally, the students who fear to meet the engineering admission requirements in reputed colleges or due to their family's economic background opt for the programs of polytechnic colleges after completing 10th standard in their schools. This is expected to get them a quick job offer in industries at shop floor or supervisory positions with a relatively lower salary compared to graduate engineers. To give them a chance to enhance their career prospects, the diploma holders were allowed to join the engineering degree program in the first year along with the regular students who join after higher secondary courses. However from the year 2004, diploma holders were permitted to join directly in the second year of engineering under the LE scheme. Initially 10 percent extra capacity over and above the approved intake for regular program was allowed for accommodating the diploma students ('Tamilnadu Government Order', 2004). Later in the year 2011, a further increase was allowed by

Revised Manuscript Received on 14 August, 2019.

G.S.Nandakumar, Department of Information Technology, Kumaraguru College of Technology, Coimbatore, Tamilnadu, India.(Email: nandakumar.gs.cse@kct.ac.in)

L.M.Palanivelu, Consultant, FIE Member (Email: palanivelulm@gmail.com) enabling engineering institutions to admit 20 percent over and above the approved intake (All India Council for Technical Education [2], [6], [3]. The increased class strength along with the differences in the backgrounds of LE students has definite impact on the quality of the programs.

\section{OBSERVATIONS DURING THE PROGRAM}

Courses in basic sciences offered in the first year are missed by the LE students who find several subjects in second and third years difficult to digest resulting in disproportionate failure rates in them. Though short bridge programs are offered to these students in individual colleges, they are unable to overcome the deficiencies effectively.

The emphasis on theoretical grasp is much lower during polytechnic education and hence LE students find it difficult to compete with regular students. The language used in the class room also creates barrier for students from rural polytechnics where they are mostly taught in vernacular though formally in a program with English medium. Entering midstream, they also find it difficult to socialize comfortably with the regular stream students. Feedback from some of the lateral entry students convey that they were misled to believe that studying engineering after diploma would be easy [7].

\section{PERFORMANCE IN EXAMINATIONS \& PLACEMENTS}

The failure rate of LE students in the early semesters of the degree programs, especially in analytically oriented subjects, are found to be high. These arrears often accumulate with repeated failures in subsequent attempts to clear them until they reach their final year. Most of the campus placement opportunities in the final year are denied to the students with standing (uncleared) arrear subject. This results in the proportion of eligible LE students for placement entry tests far lower than that of students in the regular stream as shown in Fig.1.

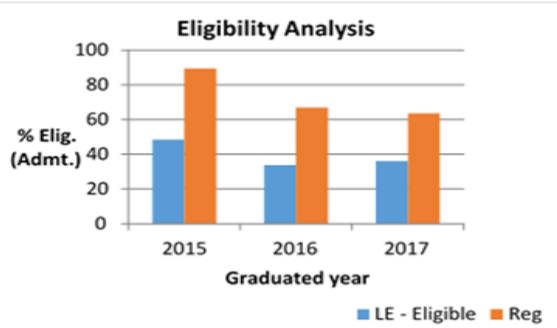

Fig.1 : The graph of students become eligible for placement year wise 


\section{A RESEARCH ON THE PERFORMANCE OF THE DIPLOMA STUDENTS IN THE LATERAL ENTRY SCHEME OF ENGINEERING DEGREE PROGRAM}

Even among the students appearing for the entrance test and subsequent interview, the percentage of LE students selected for placements is significantly lower than that for regular stream students as shown in Fig.2.

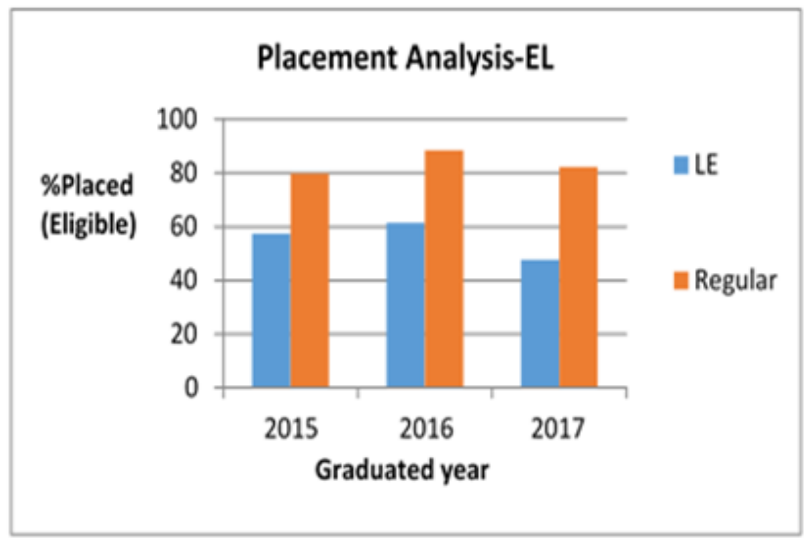

Fig. 2: The graph of students (eligible) placed year wise

Out of the total admitted students, the overall placement ratio of regular stream as well as LE students is shown in Fig.3. The trend clearly shows that the chances of an LE student getting placed are roughly one third of a regular student. This glaring difference impacts on the self confidence and psychological makeup of some LE students.

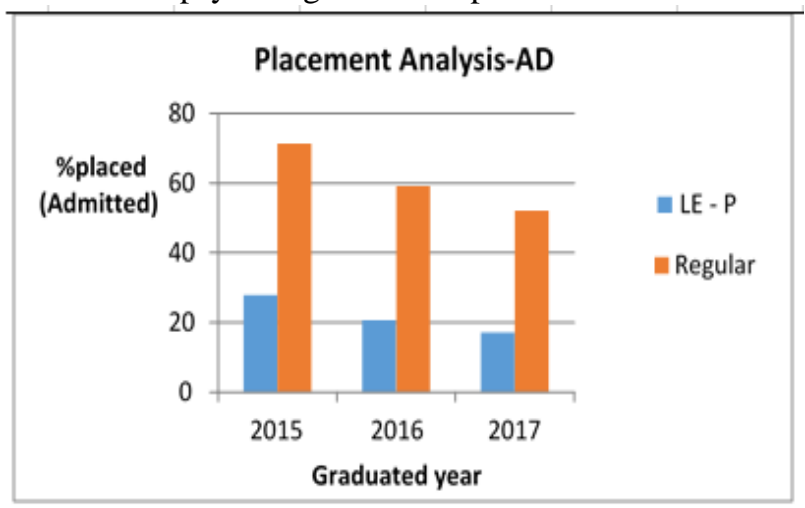

Fig. 3 The graph of students (admitted) placed year wise

\section{FACTORS CONTRIBUTING TO THE UNDERPERFORMANCE OF THE LE STUDENTS}

- $\quad$ The LE entry students are admitted to engineering colleges of choice based on their final percentage marks in Diploma programs through counseling. AICTE had instituted an entrance test to be conducted by admitting colleges as a prerequisite to the direct second year admission to the engineering education and students have to be selected only based on the merit [1], [4]. However for maximum utilization of LE seats, the colleges silently refrain from conducting such entrance test.

- $\quad$ Frequently the lateral entry students join weeks after the start of academic sessions for regular second year students. There is no separate counseling given to LE students at the time of admission or later to socialize and improve their communication skills.

\section{SUGGESTIONS FOR IMPROVING THE LE SCHEME\& RESULTS}

Revert back to the initial method of allowing selected diploma holders to join degree programs from the first year.
A specified quota of seats may be allotted during counseling for regular admissions. The extra one year spent will be more than compensated by a far improved employability by successful exploitation of their exposure to the domain during the diploma program.

- If the current LE program needs to be continued,

- admit only after a suitable qualifying entrance test [5].

- option to admit in second year should be based on the successful clearance of the entrance test criteria.

- identify pre-requisite courses with additional credits assigned to them

- synchronize LE admissions in phase with the start of third semester

- impart counseling to LE students enabling better interaction and socialization with peers

\section{CONCLUSION}

It is clear from this study that presently many LE students in engineering trail behind regular students and find it difficult to face the competition. It is time for the authorities to relook into this LE scheme and take appropriate measures so that all the stake holders' interests are better served..

\section{REFERENCES}

1. All India Council for Technical Education (AICTE) Notification 2007 retrieved 28 January 2019 from https://www.aicteindia.org/downloads/NotificationAdmis sionThroughLateralEntryRegulations2007.PDF

2. AICTE Notification 2010 retrieved 24 January 2019 from https://www.aicteindia.org/downloads/Regulations_for_approval_Process_ 2011-12.PDF

3. AICTE Approval Process Handbook 2018-19 2018 retrieved 24 January 2019 from https://www.aicteindia.org/bureaus/approval/ap_2018-19

4. Department of Technical Education, 2015, 22 April Letter No 3700/J2/2015 retrieved 24 January 2019 from https://www.svce.ac.in/admissions/le-guidelines.pdf

5. Government of Tamilnadu 2004 Higher Education Department (G.O.Ms.No.228) retrieved 30 May 2018 from http://www.tn.gov.in/exwel/gos/go_e_228_04.pdf

6. Government of West Bengal 2012 Department of Higher Education retrieved 24 January 242019 from https://wbxpress.com/admission-through-lateral-entryscheme/

7. The Hindu, 2007, January 07 Is the lateral entry system lateral in the real sense? Retrieved 24 January 2019 from http://www.thehindu.com/news/cities/Coimbatore/is-thelateral-entry-system-lateral-in-the-realsense/article4282215.ece. 